\title{
The MeerKAT Max-Planck S-band System
}

\author{
M. Kramer*, K. Menten, E.D. Barr, R. Karuppusamy, C. Kasemann, B. Klein, E. Ros, \\ G. Wieching, O. Wucknitz
}

MPI für Radioastronomie, Bonn, Germany

E-mail: mkramerampifr.de

\begin{abstract}
The science potential of MeerKAT is enormous. In order to complement existing instrumentation and with the aim to help exploiting the new instrument in an optimal way, the MPIfR is providing a complete receiving system at "S-band", i.e. 1.75-3.50 GHz.. The Max-Plank S-Band System (MP-SbS) will especially enable science that falls into the core interests of the MPIfR. These include fundamental physics with tests of theories of gravity and gravitational wave detection, the exploration of the dynamic radio sky (e.g. by detecting fast cosmological radio bursts), highly sensitive molecular spectroscopy of the interstellar medium, polarisation continuum studies, and high-resolution imaging of radio sources using very-long-baseline-interferometry (VLBI).

In addition to providing the frontend, the MP-SbS includes a state-of-the-art digital backend system that consists of a beamformer, a storage facility and a powerful compute cluster that allows turning MeerKAT into a discovery machine for pulsars and other time-domain phenomena. The system will be designed and constructed by the MPIfR in close collaboration with colleagues from the Universities of Manchester and Oxford. A major important contribution comes from the ERC Advanced Grant project MeerTRAP (PI Stappers) that will allow to double the initially planned beam former capabilities to 400 beams, although the final implementation may allow many more beams than that. Descriptions of MeerTRAP and the related MeerKAT projects TRAPUM and MeerTime can be found elsewhere in these proceedings.
\end{abstract}

MeerKAT Science: On the Pathway to the SKA

25-27 May, 2016

Stellenbosch, South Africa

${ }^{*}$ Speaker. 


\section{Introduction}

Radio astronomy is experiencing an important period of change, enabled by the revolution in digital electronics and affordable computational capability. This revolution enables the digitization, combination and processing of large bandwidths of signals. Therefore, one is able to construct sensitive telescopes out of a large number of smaller dishes, combining sensitivity with image quality, large bandwidth and high resolution. At the same time, one aims for mass production of technical elements to reduce costs, where the costs of multiplied instruments has to be balanced with the costs for increased required computing power. For this reason, we can expect that there is always a need for both interferometers and large single dishes, but it is also clear that the largest sensitivity will eventually be achieved by using arrays of telescopes, which can either work as interferometers for imaging and as a phased-array for time-domain processing, or as a station in a global network for very-long-baseline-interferometry (VLBI). The ultimate telescope pursuing this strategy is, of course, the Square Kilometre Array (SKA), but MeerKAT will already demonstrate the SKA's promises.

When completed, MeerKAT will be, by far, the most sensitive radio telescope in the Southern hemisphere. The science potential of the telescope is therefore enormous. Recently, the MPIfR, supported by funds from the Max Planck Society, embarked on a plan to support the South African activities by contributing directly to MeerKAT, choosing to provide additional capabilities to the telescope that centre on provision of an observing band at 1.75 to $3.50 \mathrm{GHz}$ ("S-band"). This particular choice was driven by a number of criteria:

Science: In many respects and for many projects, S-band is an ideal frequency, including for a pulsar survey in the inner Galaxy, high precision timing, spectroscopy $(\mathrm{CH})$, magnetism and VLBI. We present some of these ideas in more detail below.

Challenges: Especially for pulsar surveys, the lower flux density at higher frequencies needs to be compensated for by using larger bandwidths. At the same time, the smaller telescope beams require a larger number of synthesised beams to achieve a reasonable survey speed. Overall, this leads to larger requirements in data transport and processing.

Technology: The realisation of the project requires the application of a number of state-of-the-art technologies at the same time, including wide-band single pixel feed receivers, direct signal digitization without causing RFI, large-scale beamforming, data transport and analysis.

In the following we provide details of the science case before describing the instrumentation provided by the MP-SbS project.

\section{Science Cases}

The science enabled by the MP-SbS is wide ranging. Here, we concentrate on some opportunities that lie within the core interests of the MPIfR science activities. 


\subsection{Pulsars \& Transients in the dynamic radio sky}

There are not many astronomical objects whose discovery and subsequent studies provide more insight into such a rich variety of physics and astrophysics than pulsars. Applications of pulsars include using them as probes for the structure and properties of the Milky Way, the evolution and death of massive stars, their interaction with binary companions, as laboratories for physics under extreme conditions, or their use as cosmic clocks in timing experiments for testing theories of gravity. In almost every aspect, the extreme conditions in and around pulsars, including extreme magnetic and gravitational fields, exceed conditions that can be probed in terrestrial laboratories or even in the solar system. Ample examples show that discovering more of those sources leads to new and potentially transformational science. This is the motivation for the TRAPUM project, which is described in detail in the corresponding contribution in these proceedings. Referring to the TRAPUM contribution for more in-depth information, in summary, the TRAPUM aims include:

- Increase the sample sizes of all types of radio emitting neutron stars to constrain the birth rates, and the spatial and velocity distributions. This is the key for understanding the total numbers of neutron stars, the relationships between different populations and their formation rates;

- Study the properties, dynamics, and evolutionary history of globular clusters by finding new pulsars and fast transients associated with them. The new pulsars will include exotic systems, which can be used, among other things, to determine neutron star masses;

- Investigate the dependence of the pulsar and fast transient populations on properties such as metallicity and star formation history, by searching for them in external galaxies;

- Discover pulsars in the Galactic Centre and use them to probe gravitational effects of Sgr $\mathrm{A}^{*}$, the supermassive black hole at the centre of the Milky Way, dark matter and stellar populations;

- Discover exotic systems containing pulsars and compact companions and new millisecond pulsars (MSPs) to enable improved tests of general relativity and facilitate the search for gravitational waves;

- Expand the number of transient radio sources such as RRATs, giant pulses, flare stars and active binaries.

- Greatly expand the parameter space: sensitivity; search volume; time resolution, searched for fast transient radio emitters. Thereby enabling the study of the highest energy density events in the Universe and potentially identify electromagnetic counterparts to gravitational wave emission events;

- Search for radio bursts potentially associated with events at high redshift and use them for cosmology.

MeerKAT has the sensitivity to make significant contributions to all those areas. With the increasing exposure to Radio Frequency Interference (RFI) everywhere else in the world, the legallyprotected radio-quiet site of MeerKAT may also be one of a few places in the world where this is 
possible. However, enhancement of its processing capabilities are needed to provide the field-ofview that is necessary to reach the full potential of the science proposed here. Indeed, surveying the sky for pulsars and fast transients with MeerKAT will require the simultaneous creation of initially tens and ultimately hundreds of tied-array beams. This is a critical and essential aspect, both for the online creation of the beams themselves and the subsequent offline scientific processing of them. Learning how to do these types of searches will be a critical aspect of preparing for similar observations with the SKA. For this reason, MP-SbS will not only provide the receivers themselves, but in collaboration with colleagues at the Universities of Manchester and Oxford, we will develop and implement the required backend system. This is in particular possible by combining MPIfR resources with those of MeerTRAP, provided by an ERC Advanced Grant to PI Stappers.

\subsubsection{Pulsar searching}

As pulsars are Galactic objects, most of them can be found from the Southern hemisphere. This explains why the relatively small 64-m Parkes telescope has discovered about $2 / 3$ of all known pulsars. Key was the exploitation of a 13-beam receiver, which increased the telescope's survey speed by an order of magnitude. It is therefore no surprise that essentially all state-of-the-art surveys utilise multi-beam systems. The largest effort is represented by the High Time Resolution Universe Survey (HTRU), whose Southern part was done with the 13-beam system on Parkes [1], while the Northern complement is done with the 7-beam system at the 100-m telescope at Effelsberg [2]. Both survey parts utilize a search frequency around $1.4 \mathrm{GHz}$ and a bandwidth of about $300 \mathrm{MHz}$. While being highly successful, the current surveys at or below $1.4 \mathrm{GHz}$ are limited in their search volume by interstellar scattering, i.e. a pulse broadening due to multipath propagation in the interstellar medium that cannot be removed by instrumental means (unlike interstellar dispersion, which can be compensated by high frequency resolution). Scattering smears the pulse and renders it undetectable, if the scatter broadening time becomes comparable to the pulse period. This fact limits the discovery of millisecond pulsars at $1.35 \mathrm{GHz}$ (HTRU-S) to a typical distance of about $5 \mathrm{kpc}$ towards the Galactic Centre [1]. However, scatter broadening reduces as a strong function of frequency $\left(\tau \propto v^{-4.4},[3]\right)$. Hence, moving the band centre from 1.35 $\mathrm{GHz}$ to $1.75 \mathrm{GHz}$, or even $3.3 \mathrm{GHz}$, would reduce the scattering time from $5 \mathrm{~ms}$ at $5 \mathrm{kpc}$ to $1.6 \mathrm{~ms}$ and only $0.1 \mathrm{~ms}$, respectively. While this "clearing-of-the-fog" vastly extends the scattering-limited search volume, making the inner Galaxy accessible for fast-spinning pulsars for the first time, the drawback is the reduced flux density due to an average steep spectrum of pulsars $\left(S \propto v^{-1.6}\right.$, [3]). At the same time, the beam width of a radio telescope also reduces with increasing frequency $\left(\theta \propto v^{-1}\right)$, so that in the past, covering the same area of the sky, resulted typically in reduced observing time per pointing and hence reduced sensitivity.

For TRAPUM we plan to use mostly the standard 1.4-GHz frequency, as this was provisioned for in the planned MeerKAT roll-out. However, based on our recent HTRU results [4], a sensitive survey at slightly higher frequencies is paramount to probe deeper. However, such survey is impossible to perform at Parkes, or any other current large radio telescope due to the small telescope beams. Implementing a survey capability for 1.75 to $3.5 \mathrm{GHz}$ on MeerKAT will change this dramatically. Key is MeerKAT's large improvement in telescope gain and the implementation of a beam former that will produce at least 400 beams. Thanks to a combination of remarkable 
sensitivity and FoV, a corresponding extension of the TRAPUM survey programme will be hugely successful.

The strategy for an S-band survey will be finalized after on-going observations of the inner Galaxy using other telescopes. Currently, we intend to search a narrow strip given by $|l|<30 \mathrm{deg}$ and $|b|<0.5$ deg with an integration time of about $5400 \mathrm{~s}$. Based on simulations of the expected yield, we can expect the discovery of up 750 new normal and a few hundred new millisecond pulsars. These numbers are based on the initially available bandwidth of $875 \mathrm{MHz}$, so that a future utilisation of the full band will lead to even larger yields. Apart from greatly enhancing our knowledge of the Galactic population of pulsars and the usage of the new sources to probe and map the interstellar medium, this large yield of pulsars will also harbour new and exciting systems for testing theories of gravity and objects that may push the extreme of neutron star properties in terms of spin period and masses, with all the ramifications for fundamental physics that we have demonstrated successfully in the past.

In addition to the large area surveys described above, MeerKAT will also be a superb telescope for targeted searches. As shown in the past [5], the proposed frequencies range is an ideal choice to find pulsars in globular clusters. Such high-frequencies searches have for instance been used successfully to find the fastest-known spinning pulsar [6] or to find a radio magnetar in the Galactic Centre [7]. In particular, the latter discovery made with the Effelsberg telescope suggests that it is possible to find normal pulsars around the central mass black hole, known as Sgr A*, which will provide a unique probe for the black hole properties and their description in theories of gravity [8].

\subsubsection{Fast Radio Bursts}

Pulsar surveys are not only sensitive to periodic signals, but the same data also allow the discovery of single radio transients. This was demonstrated by the discovery of fast radio bursts (FRBs, [9]) and the confirmation of the existence of a whole population of cosmological transient signals using HTRU data [10]. FRBs are single broadband bursts of radio emission, with duration of a few milliseconds, that appear to represent a new class of astrophysical object, with a dozen or so examples now known. Our current estimates, based on the discovery of FRBs at Parkes and recently Arecibo and GBT, suggest that there are as many as $\sim 8,000$ FRBs per sky per day, i.e. one FRB every 10 seconds or so [11], while one has also been observed to repeat [12]. We know that they originate at cosmological distances, based on their dispersion and scattering properties. Hence, FRBs are of interest for two main reasons:

1. The progenitors are completely unknown: due to the large FRB (radio) luminosity the mechanism must be much more energetic than that of pulsars, exceeding the typical pulse luminosity by three orders of magnitude. In order to solve the mystery of their progenitors and to answer the question as to whether they are "radio-only" events, identification of their host galaxies and independent redshift estimates are needed. These require accurate localisation to perform effective multi-wavelength follow-up that would be enabled by the planned online detection pipeline which will be be implemented as part of the MeerTRAP project (PI Stappers).

2. As FRBs are detectable out to cosmological distances, they represent perfect tools to study the intergalactic medium (IGM) and probe cosmology, e.g. the detection of the rotation 
measure of an FRB can be used to measure the magnetic field. Moreover, the dispersion of these pulses is caused by the low-density, high-temperature component of the ionised IGM, impossible to detect otherwise, possibly representing "the missing baryons", where we can use the observed dispersion to "weigh" these particles [13]. Furthermore, if FRBs prove to be standard (or standardisable) candles, they can be used to measure cosmological parameters such as is done with Type Ia supernovae (see [14] and references therein). Consequently, TRAPUM and MeerTRAP will lead to the discovery of new FRBs, and combined with the multi-beaming and imaging capability of MeerKAT, new insight in the origin of FRBs and their usage as IGM probes.

\subsubsection{Pulsar timing: theories of gravity and gravitational wave detection}

Extracting the science from pulsar discoveries requires the regular monitoring of the received pulses in a technique known as "pulsar timing". Registering pulse times-of-arrival (ToA) allows a range of experiments that include tests of theories of gravity or the detection of low-frequency gravitational waves (GWs). For the detection of GWs, our aim is to measure small variations in the ToAs that are caused by the modification of space-time due to the passing of a GW over the Earthpulsar system. Acting as a cosmological GW detector, an array of timed pulsars (a so-called "pulsar timing array" or PTA) will be sensitive to nano-Hertz frequencies, thereby complementing efforts to detect $\mathrm{GW}$ at $\mathrm{kHz}$-frequencies with ground-based detectors and $\mathrm{mHz}$-frequencies with space-born detectors. Such efforts are ongoing in Australia using Parkes, in North America using the Arecibo and Green Bank Telescope, and in Europe using the 100-m Effelsberg telescope and the telescopes at Jodrell Bank, Westerbork, Nancay and Sardinia. Combining the five European telescopes to form the Large European Array for Pulsars (LEAP, ERC Advanced Grant, PI: Kramer [15, 16]) results in unprecedented sensitivity and hence timing precision across the sky, but a major difficulty for all PTA experiments is the effect of interstellar weather: tiny variations of the dispersion measure (DM) of the order of $0.1 \%$ or less lead to significant variations in the ToAs, whose red-noise properties of the resulting signal make the detection of GWs difficult. Apart from multi-frequency observations to measure and remove the effect of DM variation, a most valuable approach is to perform high precision timing at a higher radio frequency as the ToA variations scale with $(\Delta t \propto$ $\left.D M / v^{2}[3]\right)$. With the proposed receiver system, we can not only perform high-frequency timing observations that will serve as a baseline for the on-going PTA experiments, but the large sensitivity of MeerKAT promises to produce the best possible ToA measurements, complementing our efforts at Effelsberg and the other European telescopes in the Northern hemisphere to finally allow a direct GW detection.

By probing much deeper into the Galaxy with the TRAPUM survey, we will also discover many more fast-spinning objects and compact binary systems. By using the precise clock-like nature of pulsars and their role as compact test masses, we can use pulsar timing to compare their orbital motion with predictions of general relativity and alternative theories of gravity, enabling tests in strong gravitational fields. For instance the Double Pulsar provides the best test of GR in the strong field regime [17], while relativistic white dwarf-pulsar systems (WD-PSRs) like PSR J0348+0432 provide the most stringent limit on alternative theories and constraints on the equationof-state of super-dense matter [18]. The ultimate goal is the discovery and exploitation of a pulsarblack hole binary. TRAPUM is not only an excellent way of finding these systems, but it will be 
invaluable in also constraining their total number with all its ramifications for population synthesis studies and predictions for Advanced LIGO detection rates.

\subsection{A Renaissance of Radio Observations of the Molecular Diffuse Interstellar Medium}

The methylidyne radical $(\mathrm{CH})$ was the first molecule found in interstellar space. Three optical absorption lines near $400 \mathrm{~nm}$ wavelength were first found by Dunham in 1937 [19] and became a common probe of the diffuse interstellar medium in the Solar neighbourhood. At radio wavelengths, the three hyperfine structure (hfs) transitions arising between energy levels of CH's rotational ground state at $3.264,3.335$ and $3.349 \mathrm{GHz}$ were first detected at the Onsala Space Observatory [20]. The ensuing extensive surveys established $\mathrm{CH}$ as an ubiquitous component of the interstellar medium, Galaxy wide; see, e.g., [21, 22].

It readily was realized that this molecule's excitation was inconsistent with the assumption of local thermal equilibrium (LTE) since its lines were always found in emission, even toward strong continuum sources. This (generally weak) maser emission complicates the interpretation of $\mathrm{CH}$ radio observations and renders abundance observations uncertain. This and the emergence of large-scale studies of millimetre-wavelength emission from carbon monoxide contributed to a general dismissal of the $\mathrm{CH}$ radio lines as a tracer of the ISM. Nevertheless, a series of studies presented it as a useful probe of diffuse molecular material in translucent clouds; e.g., [23].

Over the last few years, the appreciation of $\mathrm{CH}$ as a tracer of diffuse interstellar clouds, i.e., of their molecular hydrogen $\left(\mathrm{H}_{2}\right)$ content, has dramatically changed with observations of absorption in the molecule's 533/537 GHz rotational submillimetre-wavelength (submm) ground-state transitions using the Heterodyne Instrument for the Far-Infrared (HIFI), the high resolution spectrometer aboard the Herschel Space Observatory [24, 25]. Even more recently, CH's 2007 GHz fine structure transition, which also connects to the molecule's ground state has become observable with GREAT, the German Receiver for Astronomy at Terahertz frequencies [26], on board the Stratospheric Observatory for Infrared Wavelengths (SOFIA). $\mathrm{H}_{2}$ itself, by far the most abundant species in the molecular ISM, has no easily observable spectral lines. Therefore, its column density and thus an interstellar cloud's mass is generally probed by the emission or absorption of much less abundant "tracer" molecules, often rare isotopologues like $\mathrm{H}^{13} \mathrm{CO}^{+}$, which each have their virtues and drawbacks. The important recent finding, also based on HIFI/Herschel data, that an unknown but significant percentage of all interstellar molecular gas is not traced by $\mathrm{CO}$ at all, but by the 158 $\mu \mathrm{m}$ fine structure line of ionized carbon (CII) [27] make observations of $\mathrm{OH}$ and $\mathrm{CH}$ all the more interesting as potential tracers of this so-called "CO-dark" molecular gas [28].

With the Herschel mission ended and SOFIA observations being expensive and only possible for a limited number of sources, the question of further extensive observations of the useful $\mathrm{CH}$ molecule has arisen. The sub-mm lines cannot be observed from the ground because of the Earth's atmosphere. In contrast, the three radio lines around $3.3 \mathrm{GHz}$ can be observed very well, for example with the Effelsberg 100-m telescope and, recently, even imaged with the newly extended Jansky Very Large Array and in the future with MeerKAT. As explained above, the $3.3 \mathrm{GHz} \mathrm{CH}$ hfs lines are always inverted which makes their interpretation difficult. This can be remedied once collisional rate coefficients have become available that allow non-LTE excitation/radiative transfer modeling of the molecule's excitation using the measured intensities of the sub-mm/far infrared and all three radio hfs lines as constraints. Experts in the determination of such coefficients are 
currently engaged in the necessary quantum mechanical calculations. With such rate coefficients in hand, one could use the HIFI and SOFIA sub-mm/far infrared wavelength absorption spectra for the few sources for which such data are available, to "calibrate" the information provided by the radio lines and in the end hopefully use the radio lines alone to determine $\mathrm{CH}$ column densities for many lines of sight.

Given all this, we are convinced that the time has come for the $3.3 \mathrm{GHz} \mathrm{CH}$ lines to become highly useful probes of the ISM throughout the Milky Way, along with the hfs lines of hydroxyl $(\mathrm{OH})$ near $1.7 \mathrm{GHz}$, which will also be observable with MeerKAT. The frequency extension of MeerKAT enabled by MP-SbS will allow imaging of the three $3.3 \mathrm{GHz}$ lines of $\mathrm{CH}$ throughout the Southern galaxy and even in extragalactic sources. It will add crucial information on the diffuse ISM that will be highly complementary to the comprehensive surveys of the denser "CO-bright" ISM that the Atacama Pathfinder Experiment Telescope (APEX) is delivering [29, 30]. Like its sub-mm absorption, CH's radio emission is seen toward distant sources of continuum radiation from star forming regions. Thus the $\mathrm{CH} 3.3 \mathrm{GHz}$ lines, with the information they carry calibrated as described above, will probe the $\mathrm{H}_{2}$ column density of all the intervening spiral arms on the lines of sight to distant background sources, delivering pencil beam "tomography" of the molecular ISM of large parts of the Milky Way and even nearby galaxies, in particular the Magellanic clouds.

\subsection{Very Long Baseline Interferometry}

VLBI provides the highest possible resolution in astronomy. It connects different radio telescopes on a regular basis for joint observations. With the MP-SbS and MeerTrap projects, MeerKAT will have beam-forming capabilities, so that it will be able to operate like a single, large telescope of equivalent diameter of over $100 \mathrm{~m}$. In this mode, it will be able to participate in regular observations of VLBI networks, substantially enhancing sensitivity. In particular, as the largest radio telescope in the Southern hemisphere, it can join the high-sensitive world-class VLBI elements such as Arecibo, Effelsberg, the phased JVLA, the Green Bank Telescope, Parkes, and the 70-m dishes of NASA/JPL's Deep Space Network (DSN). MeerKAT is located to the South-West of the 26-m dish in Hartebeesthoek (HartRAO), which is already a regular member of the European, the global, and the Australian networks. The addition of MeerKAT to these arrays will enhance the image fidelity and the Fourier sampling of radio interferometric observations dramatically.

The frequencies in the bands $\mathrm{L}(1-2 \mathrm{GHz})$ and $\mathrm{S}(2-4 \mathrm{GHz})$ are important in VLBI to resolve the optically thin, extended emission in jets of Active Galactic Nuclei (AGNs) and radio galaxies, to image supernova factories and radio supernovae during the expansion phase months or years after explosion.

Presently, the European VLBI Network (EVN), which includes VLBI stations in Europe, Puerto Rico (Arecibo) and China, operates regularly including the 26-m radio telescope in HartRAO. HartRAO is connected by optical fibre to the eVLBI facility, so that it regularly performs real-time VLBI, providing excellent North-South coverage with the Eurasian antennas and Arecibo. MeerKAT will be connected to the eVLBI network also, and it will therefore offer rapid agility for eVLBI science, such as triggered observations after transients, or regular off-session observations from the EVN in the eVLBI slots. Additionally, a beam-formed MeerKAT can join observations with the Australian Long Baseline Array, enhancing the Southern network at decimeter wavelengths, exceeding Parkes in sensitivity for long and short baselines to New Zealand. 
Table 1: Frontend specifications, if applicable given for $95 \%$ of the band

\begin{tabular}{ll}
\hline Number of Rx & 2 prototypes (spares) +64 production versions \\
Sky frequency & $1.75-3.50 \mathrm{GHz}$ \\
Noise temperature: & \\
(Trec+Tspill) & $<25$ Kelvin \\
Tsys & $<32$ Kelvin \\
Polarisation & $H \& V$, cross coupling $<20 \mathrm{~dB}$ \\
Stability & $>1000 \mathrm{sec}$ spec Allan variance (@ $1 \mathrm{MHz}$ bandwidth) \\
\hline
\end{tabular}

Besides being more sensitive, the addition of a new antenna in the VLBI arrays at a distance of $900 \mathrm{~km}$ (namely, 5-7 M $\lambda$ at this wavelength range) from HartRAO is essential for a better image fidelity for extended emission and for the mutual short-baseline calibration with HartRAO in arrays both towards Australia or Europe. This has direct impact, for instance, on the quality of high-resolution images of the lobes of nearby radio galaxies such as Centaurus A or Cygnus A. The ongoing build-up of an African VLBI Array including telescopes in Ghana, Kenya, and Madagascar, promises the future availability of a powerful African-wide array further enhance the global VLBI network with MeerKAT at the core of it.

\section{System Description}

The MP-SbS receiver will be installed on the MeerKAT antennas. For this purpose the antenna has been equipped with an instrument indexer for remote selection of up to four different receivers. To reduce installation efforts and increase possible support from the MeerKAT team we intend to follow the interfaces of the native MeerKAT L-Band receiver. This includes, but is not limited to, vacuum and $\mathrm{He}$, and power supply as well as data transfer and control infrastructure. In addition significant parts of the existing MeerKAT backend structure will support the MPIfR data reduction.

\subsection{Frontend description}

Each of the 64 MeerKAT antennas will be equipped with one receiver frontend. The receiver frontend includes all components to collect, amplify and digitize the signal. The frontend transmits the digitized signals via the MeerKAT communication network, using standard Ethernet, to the backend section of MeerKAT.

Even though the interfaces to the MeerKAT infrastructure mostly follow the interfaces defined by the L-Band receiver of MeerKAT, the MPIfR receiver design reuses, if applicable, developments that have already been made and tested at the MPIfR. Thus the design for the MeerKAT receiver benefits significantly from previous projects carried out by the technical department of the MPIfR. This does not only reduce the development requirements, but also, more importantly, decreases the risks, during the design as well as the operations, as the existing hardware parts are thoroughly tested, some even for several years at telescope environments. The frontend specifications, if applicable given for $95 \%$ of the band, are presented in Table 1, which summarizes the performance of the frontend, required to enable the science presented above. 
Note that the data will be digitized right at the front-end, where a electrical and spatial separation of ADC and FPGA is implemented to reduce RFI. The ADC will be integrated in the receiver package, providing direct sampling with $1.75 \mathrm{GHz}$ bandwidth and 12 bits. The data are repacked to achieve an internal data reduction to $875 \mathrm{MHz}$ at 10Bit precision, in order to be compatible with MeerKAT's standard backend system. Each data stream from the receiver will receive absolute time stamping.

\subsection{Backend system}

For the spectroscopy and imaging applications, the MeerKAT correlator will be able to perform the required tasks. For VLBI and in particular the non-imaging (time-domain) processing, it is necessary to design and deploy a beam former whose output will be recorded and processed by a computing/storage cluster. For the transient science case, it is important that FRBs will be detected in quasi-real time in order to provide triggers for observatories at other parts of the electromagnetic spectrum. Moreover, looking ahead, the data rate for SKA Phase 1 will be so high, that all pulsar searching has to be implemented in an on-line system. The development and verification of such system is extremely important. Also here, MeerKAT serves as an important stepping (and verification) stone, where we can implement an on-line search system, whose output can be compared to a later off-line processing using the stored data. In the following we describe the components needed.

\subsubsection{Beamformer}

The scientific goal of the pulsar survey is to enable the search of the entire sky visible from MeerKAT for pulsars. As MeerKAT consists of an array of telescopes, obtaining the maximum sensitivity requires adding the signals of these elements coherently. This results in a narrow beam on the sky, and thus in a very slow survey speed. To compensate for that, a large number of beams are formed to dramatically speed up the survey. In the case of MeerKAT, together with MeerTRAP, we aim for a compromise between survey speed and computing power, resulting in at least 400 beams. Advances in compute power since the inception of the system mean that we will probably be able to produce more than 1000 beams eventually. This is comparable with the SKA1 requirement and hence provides an excellent testbed.

During the beam forming process, signal streams from different antennas are combined with appropriated phase delays to single out certain beam directions within the primary field-of-view of the single MeerKAT antenna. This is effectively done in the frequency domain using a GPU implementation.

\subsubsection{Storage cluster}

The output of the beam former will be (at least) 400 beams with 4096 channels, 8 bits and a sampling time of $64 \mu \mathrm{s}$. This results in a data rate of about $64 \mathrm{MB} / \mathrm{s} /$ beam or $26 \mathrm{~GB} / \mathrm{s}$ in total. These data will be transferred using 40 or 100 GigaBit-Ethernet lines via a switch to a storage cluster. While the output will be searched on-line for single pulses and FRBs within MeerTRAP, finding binary pulsars requires off-line analysis. Hence, it will be necessary to store at least one day of observing data. This results in a storage requirement of at least 2.2 PB. 


\subsubsection{Computing cluster}

The limitations in the storage capacity require an off-line processing of the stored search data, before further observations can start, as only about 1 day of searching can be recorded. The data reduction will be made using a dedicated computing cluster. As searching for pulsars is an "embarrassingly parallel" problem, it can be easily tackled in a scalable cluster, although the search for an accelerated compact binary system will be ultimately limited only by the available computing power. In order to define a minimum useful size of the cluster, we require that it is sufficiently powerful to conduct a meaningful on-line search, the results of which are then compared to the corresponding off-pulse results.

The raw data for some of the most interesting candidates are ideally kept, so that more sophisticated methods can be deployed, and so that future updated ephemerides can be re-applied to decrease any existing smearing later. For this reason, some of the data will be transferred to long-term storage. At the moment, we are exploring the possibility to use the European Data Relay System (EDRS), which communicates via optical lasers between the ground station, Low-Earth orbiting and geostationary satellites to eventually beam the data to Germany via radio down-link.

\section{Implementation Plan}

The finite lifetime of MeerKAT as an independent observatory before being absorbed by SKA, sets the early possible science utilization of the MP-SbS as a critical design goal. At the time of writing, the prototypes were successfully tested on the MeerKAT telescopes (see Figure 1 and 2). Results from this testing were used to update the design for the production frontends and the production will ramp up, for which the tests are ongoing at the time of writing. After successful completion, mass production of the receivers will commence. While most of the production receiver parts will be contracted, in order to keep control over critical parts of the receiver, we intend to perform the following tasks for the frontend in-house: LNA selection and packing, cryostat and receiver integration, and performance verification.

An test system for the beamformer and backend system is also on-site and being tested. We expect that the whole MP-SbS will be available soon after the full completion of MeerKAT in Spring/Summer 2018.

\section{Acknowledgements}

We thank our South African partners for all their support and help during the project so far, appreciating their skill, insight and high professionalism. We also thank Ben Stappers, PI of ERC MeerTRAP, and Aris Karastergiou for collaborating so closely and effectively. The system is realized in collaboration with Anton Zensus and the MPIfR VLBI group.

\section{References}

[1] M. J. Keith, A. Jameson, W. van Straten, M. Bailes, S. Johnston, M. Kramer, A. Possenti, S. D. Bates, N. D. R. Bhat, M. Burgay, S. Burke-Spolaor, N. D’Amico, L. Levin, P. L. McMahon, S. Milia and B. W. Stappers, The High Time Resolution Universe Pulsar Survey - I. System configuration and initial discoveries, MNRAS 409, 619 (2010). 

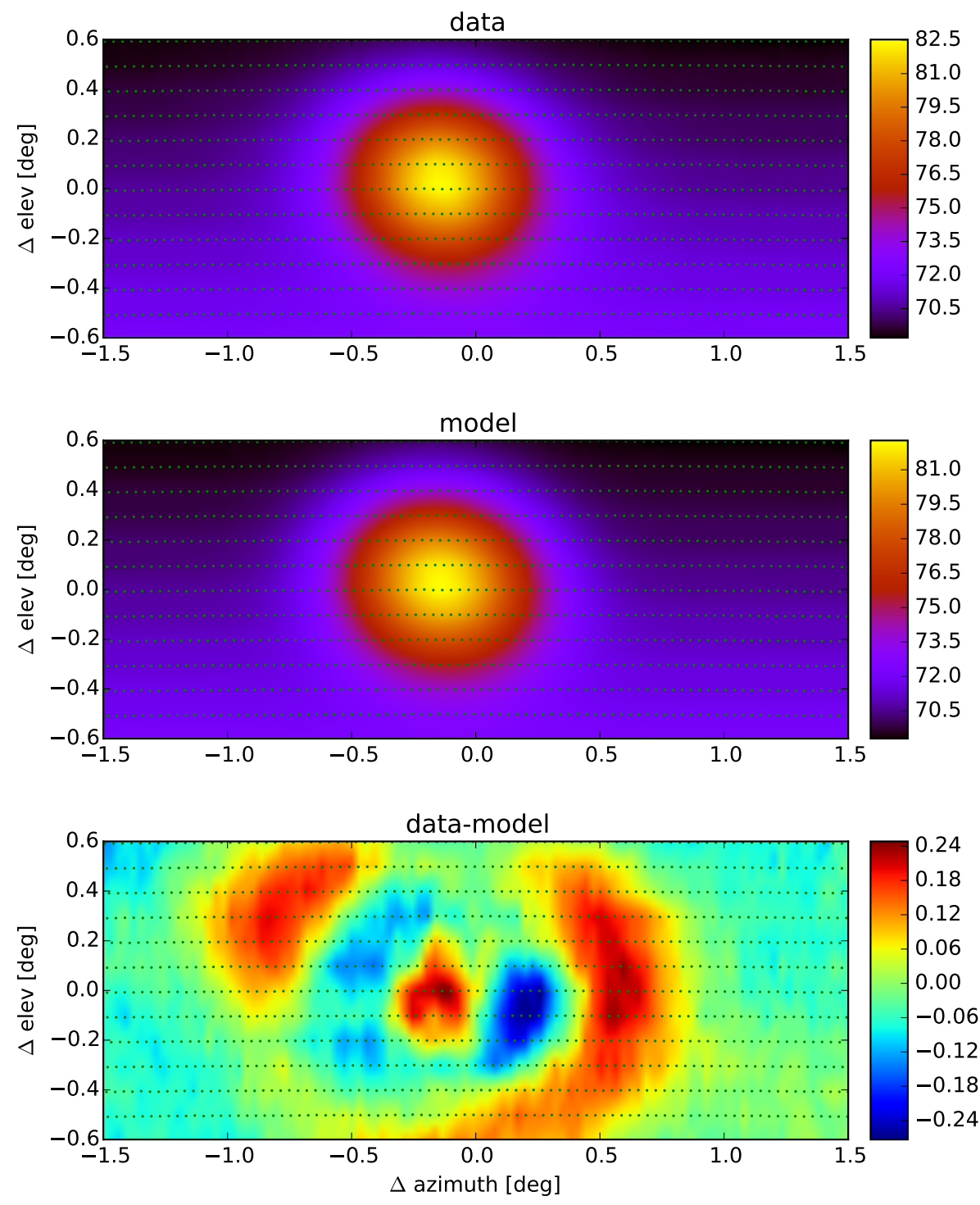

Figure 1: Frequency-averaged beam shape for one polarisation of one prototype receiver. The top panel shows the measurements (pointings denoted by green dots), the middle one an elliptical Gaussian model with noise gradient and the bottom one the residuals. Figure by Olaf Wucknitz.

[2] E. D. Barr, D. J. Champion, M. Kramer, R. P. Eatough, P. C. C. Freire, R. Karuppusamy, K. J. Lee, J. P. W. Verbiest, C. G. Bassa, A. G. Lyne, B. Stappers, D. R. Lorimer and B. Klein, The Northern High Time Resolution Universe pulsar survey - I. Setup and initial discoveries, MNRAS 435, 2234 (2013).

[3] D. R. Lorimer and M. Kramer, Handbook of Pulsar Astronomy (Cambridge University Press, 2005).

[4] C. Ng, D. J. Champion, M. Bailes, E. D. Barr, S. D. Bates, N. D. R. Bhat, M. Burgay,

S. Burke-Spolaor, C. M. L. Flynn, A. Jameson, S. Johnston, M. J. Keith, M. Kramer, L. Levin,

E. Petroff, A. Possenti, B. W. Stappers, W. van Straten, C. Tiburzi, R. P. Eatough and A. G. Lyne, The 


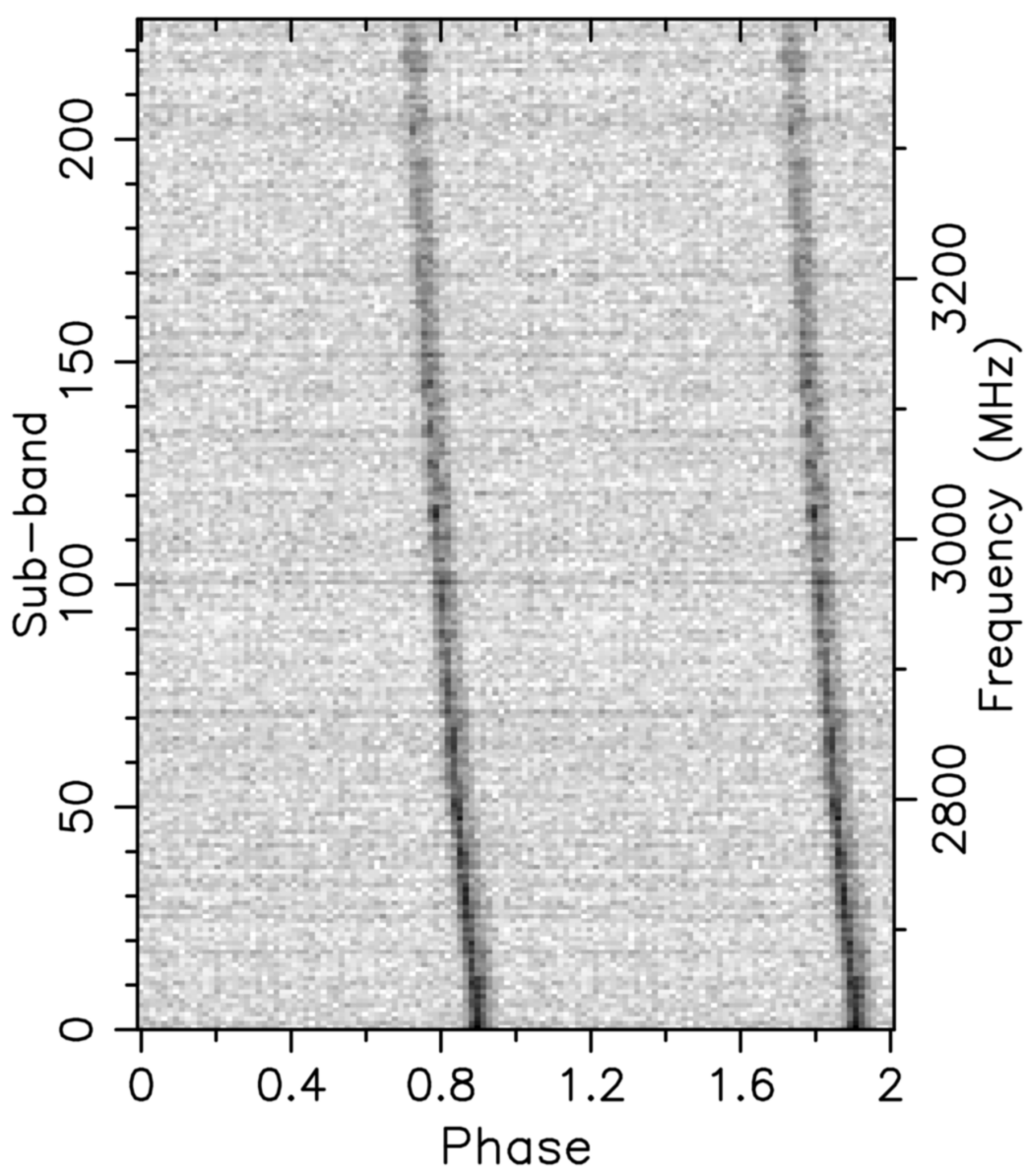

Figure 2: Pulsar observations with one of the prototype receivers. The figure shows the folded signal (two periods shown) of the Vela pulsar in the upper part of the band using only $90 \mathrm{sec}$ of one polarisation from one antenna. The interstellar dispersion has not been removed here. Figure by Olaf Wucknitz.

High Time Resolution Universe Pulsar Survey - XII. Galactic plane acceleration search and the discovery of 60 pulsars, MNRAS 450, 2922 (2015).

[5] S. M. Ransom, J. W. T. Hessels, I. H. Stairs, P. C. C. Freire, F. Camilo, V. M. Kaspi and D. L. Kaplan, Twenty-One Millisecond Pulsars in Terzan 5 Using the Green Bank Telescope, Science 307, 892 (2005).

[6] J. W. T. Hessels, S. M. Ransom, I. H. Stairs, P. C. C. Freire, V. M. Kaspi and F. Camilo, A Radio Pulsar Spinning at $716 \mathrm{~Hz}$, Science 311, 1901 (2006).

[7] R. P. Eatough, H. Falcke, R. Karuppusamy, K. J. Lee, D. J. Champion, E. F. Keane, G. Desvignes, D. H. F. M. Schnitzeler, L. G. Spitler, M. Kramer, B. Klein, C. Bassa, G. C. Bower, A. Brunthaler, I. Cognard, A. T. Deller, P. B. Demorest, P. C. C. Freire, A. Kraus, A. G. Lyne, A. Noutsos, B. Stappers and N. Wex, A strong magnetic field around the supermassive black hole at the centre of the Galaxy, Nature 501, 391 (2013). 
[8] K. Liu, N. Wex, M. Kramer, J. M. Cordes and T. J. W. Lazio, Prospects for Probing the Spacetime of Sgr A* with Pulsars, ApJ 747, p. 1 (2012).

[9] D. R. Lorimer, M. Bailes, M. A. McLaughlin, D. J. Narkevic and F. Crawford, A Bright Millisecond Radio Burst of Extragalactic Origin, Science 318, p. 777 (2007).

[10] D. Thornton, B. Stappers, M. Bailes, B. Barsdell, S. Bates, N. D. R. Bhat, M. Burgay, S. Burke-Spolaor, D. J. Champion, P. Coster, N. D’Amico, A. Jameson, S. Johnston, M. Keith, M. Kramer, L. Levin, S. Milia, C. Ng, A. Possenti and W. van Straten, A Population of Fast Radio Bursts at Cosmological Distances, Science 341, 53 (2013).

[11] D. J. Champion, E. Petroff, M. Kramer, M. J. Keith, M. Bailes, E. D. Barr, S. D. Bates, N. D. R. Bhat, M. Burgay, S. Burke-Spolaor, C. M. L. Flynn, A. Jameson, S. Johnston, C. Ng, L. Levin, A. Possenti, B. W. Stappers, W. van Straten, D. Thornton, C. Tiburzi and A. G. Lyne, Five new fast radio bursts from the HTRU high-latitude survey at Parkes: first evidence for two-component bursts, MNRAS 460, L30 (2016).

[12] L. G. Spitler, P. Scholz, J. W. T. Hessels, S. Bogdanov, A. Brazier, F. Camilo, S. Chatterjee, J. M. Cordes, F. Crawford, J. Deneva, R. D. Ferdman, P. C. C. Freire, V. M. Kaspi, P. Lazarus, R. Lynch, E. C. Madsen, M. A. McLaughlin, C. Patel, S. M. Ransom, A. Seymour, I. H. Stairs, B. W. Stappers, J. van Leeuwen and W. W. Zhu, A repeating fast radio burst, Nature 531, 202 (2016).

[13] E. F. Keane, S. Johnston, S. Bhandari, E. Barr, N. D. R. Bhat, M. Burgay, M. Caleb, C. Flynn, A. Jameson, M. Kramer, E. Petroff, A. Possenti, W. van Straten, M. Bailes, S. Burke-Spolaor, R. P. Eatough, B. W. Stappers, T. Totani, M. Honma, H. Furusawa, T. Hattori, T. Morokuma, Y. Niino, H. Sugai, T. Terai, N. Tominaga, S. Yamasaki, N. Yasuda, R. Allen, J. Cooke, J. Jencson, M. M. Kasliwal, D. L. Kaplan, S. J. Tingay, A. Williams, R. Wayth, P. Chandra, D. Perrodin, M. Berezina, M. Mickaliger and C. Bassa, The host galaxy of a fast radio burst, Nature 530, 453 (2016).

[14] J. P. Macquart, E. Keane, K. Grainge, M. McQuinn, R. Fender, J. Hessels, A. Deller, R. Bhat, R. Breton, S. Chatterjee, C. Law, D. Lorimer, E. O. Ofek, M. Pietka, L. Spitler, B. Stappers and C. Trott, Fast Transients at Cosmological Distances with the SKA, Advancing Astrophysics with the Square Kilometre Array (AASKA14) , p. 55 (2015).

[15] M. Kramer and D. J. Champion, The European Pulsar Timing Array and the Large European Array for Pulsars, Classical and Quantum Gravity 30, p. 224009 (2013).

[16] C. G. Bassa, G. H. Janssen, R. Karuppusamy, M. Kramer, K. J. Lee, K. Liu, J. McKee, D. Perrodin, M. Purver, S. Sanidas, R. Smits and B. W. Stappers, LEAP: the Large European Array for Pulsars, MNRAS 456, 2196 (2016).

[17] M. Kramer, I. H. Stairs, R. N. Manchester, M. A. McLaughlin, A. G. Lyne, R. D. Ferdman, M. Burgay, D. R. Lorimer, A. Possenti, N. D’Amico, J. M. Sarkissian, G. B. Hobbs, J. E. Reynolds, P. C. C. Freire and F. Camilo, Tests of General Relativity from Timing the Double Pulsar, Science 314, 97 (October 2006).

[18] J. Antoniadis, P. C. C. Freire, N. Wex, T. M. Tauris, R. S. Lynch, M. H. van Kerkwijk, M. Kramer, C. Bassa, V. S. Dhillon, T. Driebe, J. W. T. Hessels, V. M. Kaspi, V. I. Kondratiev, N. Langer, T. R. Marsh, M. A. McLaughlin, T. T. Pennucci, S. M. Ransom, I. H. Stairs, J. van Leeuwen, J. P. W. Verbiest and D. G. Whelan, A Massive Pulsar in a Compact Relativistic Binary, Science 340, p. 448 (2013).

[19] T. Dunham, Jr., Interstellar Neutral Potassium and Neutral Calcium, PASP 49, 26 (1937).

[20] O. E. H. Rydbeck, J. Elldér and W. M. Irvine, Radio Detection of Interstellar CH, Nature 246, 466 (1973). 
[21] O. E. H. Rydbeck, E. Kollberg, A. Hjalmarson, A. Sume, J. Ellder and W. M. Irvine, Radio observations of interstellar CH. I, 31, 333 (1976).

[22] R. Genzel, D. Downes, T. Pauls, T. L. Wilson and J. Bieging, Interstellar CH - Excitation temperatures and abundance relative to $\mathrm{H} 2 \mathrm{CO}, A \& A$ 73, 253 (1979).

[23] L. Magnani, R. J. Chastain, H. C. Kim, D. Hartmann, A. T. Truong and P. Thaddeus, CH, CO, and E(B-V) as Molecular Gas Tracers in a Translucent Cloud, ApJ 586, 1111 (2003).

[24] S.-L. Qin, P. Schilke, C. Comito, T. Möller, R. Rolffs, H. S. P. Müller, A. Belloche, K. M. Menten, D. C. Lis, T. G. Phillips, E. A. Bergin, T. A. Bell, N. R. Crockett, G. A. Blake, S. Cabrit, E. Caux, C. Ceccarelli, J. Cernicharo, F. Daniel, M.-L. Dubernet, M. Emprechtinger, P. Encrenaz, E. Falgarone, M. Gerin, T. F. Giesen, J. R. Goicoechea, P. F. Goldsmith, H. Gupta, E. Herbst, C. Joblin, D. Johnstone, W. D. Langer, S. D. Lord, S. Maret, P. G. Martin, G. J. Melnick, P. Morris, J. A. Murphy, D. A. Neufeld, V. Ossenkopf, L. Pagani, J. C. Pearson, M. Pérault, R. Plume, M. Salez, S. Schlemmer, J. Stutzki, N. Trappe, F. F. S. van der Tak, C. Vastel, S. Wang, H. W. Yorke, S. Yu, J. Zmuidzinas, A. Boogert, R. Güsten, P. Hartogh, N. Honingh, A. Karpov, J. Kooi, J.-M. Krieg, R. Schieder, M. C. Diez-Gonzalez, R. Bachiller, J. Martin-Pintado, W. Baechtold, M. Olberg, L. H. Nordh, J. L. Gill and G. Chattopadhyay, Herschel observations of EXtra-Ordinary Sources (HEXOS): detecting spiral arm clouds by $\mathrm{CH}$ absorption lines, $A \& A$ 521, p. L14 (2010).

[25] M. Gerin, M. de Luca, J. R. Goicoechea, E. Herbst, E. Falgarone, B. Godard, T. A. Bell, A. Coutens, M. Kaźmierczak, P. Sonnentrucker, J. H. Black, D. A. Neufeld, T. G. Phillips, J. Pearson, P. B. Rimmer, G. Hassel, D. C. Lis, C. Vastel, F. Boulanger, J. Cernicharo, E. Dartois, P. Encrenaz, T. Giesen, P. F. Goldsmith, H. Gupta, C. Gry, P. Hennebelle, P. Hily-Blant, C. Joblin, R. Kołos, J. Krełowski, J. Martín-Pintado, R. Monje, B. Mookerjea, M. Perault, C. Persson, R. Plume, M. Salez, M. Schmidt, J. Stutzki, D. Teyssier, S. Yu, A. Contursi, K. Menten, T. R. Geballe, S. Schlemmer, P. Morris, W. A. Hatch, M. Imram, J. S. Ward, E. Caux, R. Güsten, T. Klein, P. Roelfsema, P. Dieleman, R. Schieder, N. Honingh and J. Zmuidzinas, Interstellar CH absorption in the diffuse interstellar medium along the sight-lines to G10.6-0.4 (W31C), W49N, and W51, A\&A 521, p. L16 (October 2010).

[26] S. Heyminck, U. U. Graf, R. Güsten, J. Stutzki, H. W. Hübers and P. Hartogh, GREAT: the SOFIA high-frequency heterodyne instrument, $A \& A$ 542, p. L1 (2012).

[27] J. L. Pineda, W. D. Langer, T. Velusamy and P. F. Goldsmith, A Herschel [C ii] Galactic plane survey. I. The global distribution of ISM gas components, $A \& A$ 554, p. A103 (2013).

[28] M. G. Wolfire, D. Hollenbach and C. F. McKee, The Dark Molecular Gas, ApJ 716, 1191 (2010).

[29] R. Güsten, L. Å. Nyman, P. Schilke, K. Menten, C. Cesarsky and R. Booth, The Atacama Pathfinder EXperiment (APEX) - a new submillimeter facility for southern skies -, A\&A 454, L13 (2006).

[30] F. Schuller, K. M. Menten, Y. Contreras, F. Wyrowski, P. Schilke, L. Bronfman, T. Henning, C. M. Walmsley, H. Beuther, S. Bontemps, R. Cesaroni, L. Deharveng, G. Garay, F. Herpin, B. Lefloch, H. Linz, D. Mardones, V. Minier, S. Molinari, F. Motte, L.- $\AA$. Nyman, V. Reveret, C. Risacher, D. Russeil, N. Schneider, L. Testi, T. Troost, T. Vasyunina, M. Wienen, A. Zavagno, A. Kovacs, E. Kreysa, G. Siringo and A. Weiß, ATLASGAL - The APEX telescope large area survey of the galaxy at $870 \mu \mathrm{m}, A \& A \mathbf{5 0 4}, 415$ (2009). 